AIP Appinied Physics

\title{
Optical properties and electronic structure of polycrystalline Ag1-xCuxInSe2 alloys
}

J. G. Albornoz, R. Serna, and M. León

Citation: J. Appl. Phys. 97, 103515 (2005); doi: 10.1063/1.1899243

View online: http://dx.doi.org/10.1063/1.1899243

View Table of Contents: http://jap.aip.org/resource/1/JAPIAU/v97/i10

Published by the American Institute of Physics.

\section{Additional information on J. Appl. Phys.}

Journal Homepage: http://jap.aip.org/

Journal Information: http://jap.aip.org/about/about_the_journal

Top downloads: http://jap.aip.org/features/most_downloaded

Information for Authors: http://jap.aip.org/authors

\section{ADVERTISEMENT}

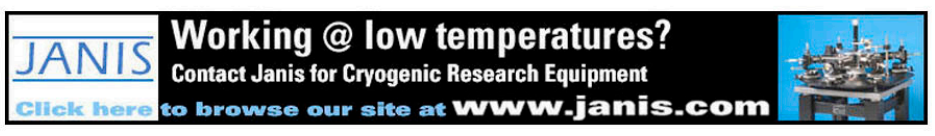




\title{
Optical properties and electronic structure of polycrystalline $\mathrm{Ag}_{1-x} \mathrm{Cu}_{x} \operatorname{lnSe} \mathrm{Se}_{2}$ alloys
}

\author{
J. G. Albornoz ${ }^{\text {a) }}$ \\ Departamento de Física, Facultad de Ciencia y Tecnología, Universidad de Carabobo, Valencia 2001, \\ Venezuela
}

\section{R. Serna}

Instituto de Óptica, C.S.I.C., Serrano 121, 28006 Madrid, Spain

M. León

Departamento de Física Aplicada, Universidad Autónoma de Madrid, 28049 Madrid, Spain

(Received 13 October 2004; accepted 9 March 2005; published online 5 May 2005)

\begin{abstract}
The dielectric function $\varepsilon(\omega)$ of polycrystalline bulk samples of the quaternary chalcopyrite semiconductors $\mathrm{Ag}_{1-x} \mathrm{Cu}_{x} \mathrm{InSe}_{2}$ with $x=0.0,0.2,0.4,0.6,0.8,1.0$ has been determined by spectroscopic ellipsometry in the energy range from 0.5 to $4.7 \mathrm{eV}$ at room temperature. Accurate values of refractive indices $n$ and extinction coefficients $k$ representative of bulk materials are obtained from the data. The value of the main energy gap is very sensitive to the composition and varies from 1.225 to $1.009 \mathrm{eV}$ as $x$ increases (Cu content). The structures observed in $\varepsilon(\omega)$ have been analyzed by fitting the numerically differentiated experimental spectrum (second derivative) to analytical line shapes. As a result, the energies corresponding to different electronic transitions have been determined as a function of the composition, and they have been identified within the electronic band structure of chalcopyrites. (C) 2005 American Institute of Physics.
\end{abstract}

[DOI: $10.1063 / 1.1899243]$

\section{INTRODUCTION}

For photovoltaic energy generation the choice of the absorber material is the key factor that ultimately determines the final efficiency that can be reached by a solar cell. In particular the accurate knowledge of the optical properties of the system is essential for the appropriate design of a high efficiency photovoltaic system. Polycrystalline thin-film solar cells based on $\mathrm{CuInSe}_{2}$ and the related quaternary alloys show remarkable physical qualities, including, among others, a high absorption coefficient and the possibility of $p$ or $n$ doping. Specifically laboratory solar cells with efficiencies as high as $19 \%$ have been demonstrated ( $\mathrm{Zn} 0 / \mathrm{CdS} /$ CuInGaSe 2 ). ${ }^{1-3}$ Quaternary alloys show the advantage of a large degree of variation of their properties as a function of the composition, which enables the tailoring of the material properties.

In this work we will focus on the quaternary system $\mathrm{Ag}_{1-x} \mathrm{Cu}_{x} \mathrm{InSe}_{2}$. This system is located between the ternary semiconducting chalcopyrite compounds $\mathrm{CuInSe}_{2}$ (CIS) ${ }^{3-8}$ and $\mathrm{AgInSe}_{2}$ (AIS). ${ }^{9-11}$ Both compounds have a high optical absorption coefficient, which is an important factor for the manufacture of devices, direct energy gap with values $E_{g}$ $\approx 1.00$ and $1.22 \mathrm{eV}$ respectively, and belong to the space group $I \overline{4} 2 d$. The CIS can be obtained with $\mathbf{p}$ - or $\mathbf{n}$-type conductivity, whereas the AIS is obtained mainly with $\mathbf{n}$-type conductivity.

Reports on $\mathrm{Ag}_{1-x} \mathrm{Cu}_{x} \mathrm{InSe}_{2}{ }^{12-19}$ and similar systems as $\mathrm{AgInS}_{x} \mathrm{Se}_{1-x}{ }^{18-20} \mathrm{Cu}_{1-x} \mathrm{Ag}_{x} \mathrm{GaSe}_{2},{ }^{20,21}$ and $\mathrm{Ag}_{x} \mathrm{Cu}_{1-x}$

\footnotetext{
a) Author to whom correspondence should be addressed; electronic mail: gregorio.albornoz@uam.es
}

$\mathrm{GaS}_{2}{ }^{22,23}$ have been mainly dedicated to the analysis of the structural parameters as a function of the composition, and a few have been focused on the electrical and optical properties. In the latter case previous studies provided the values of the band gap energies for some alloys using optical absorption deduced from reflection and transmission measurements in thin film configuration. ${ }^{12,13,23}$ Nevertheless the optical properties of $\mathrm{Ag}_{1-x} \mathrm{Cu}_{x} \mathrm{InSe}_{2}$ by spectroscopic ellipsometry have not been studied.

In this work the complex dielectric function $\varepsilon(\omega)$ of polycrystalline bulk $\mathrm{Ag}_{1-x} \mathrm{Cu}_{x} \mathrm{InSe}_{2}$ with $x=0.0,0.2,0.4,0.6$, 0.8 , and 1.0 has been determined by variable angle spectroscopic ellipsometry measurements ${ }^{24}$ in the range of energies from 0.5 to $4.7 \mathrm{eV}$, at room temperature. As a result the structures observed in the dielectric function have been analyzed and related to the electronic band structures of these polycrystalline chalcopyrites. Finally the variation of the electronic transitions as a function of the composition has been analyzed.

\section{SAMPLE PREPARATION}

The ingots of the semiconducting system $\mathrm{Ag}_{1-x} \mathrm{Cu}_{x} \mathrm{InSe}_{2}$ with $x=0.0,0.2,0.4,0.6,0.8$, and 1.0 , have been synthesized in vacuum $\left(1 \times 10^{-6}\right.$ Torr $)$ in quartz capsules, by direct fusion of the constituent elements $(\mathrm{Ag}, \mathrm{Cu}, \mathrm{In})$ previously mixed in the desired stoichiometric proportion. The composition analysis was performed by means of three different techniques: sweeping electronic microscopy (SEM-EDAX), inductive coupling plasma mass spectrometry, and x-ray fluorescence. The composition average is in good agreement with the nominal composition of the initial mixture. 
Conductivity type of the samples has been determined by means of the Seebeck effect thermal test. The ingots ${ }^{25}$ with $x \leqslant 0.4$ have $\mathbf{n}$-type conductivity, whereas the ingots with $x \geqslant 0.6$ have $\mathbf{p}$-type conductivity.

The structural analysis was performed by $\mathrm{x}$-ray measurements. The ingots were polycrystalline single phase, with chalcopyrite structure in all the studied ranges of composition. Detailed studies of structure, composition, differential thermal analysis, and electrical properties of the samples used in the present work will be the object of a separate publication. ${ }^{25}$

The samples were cut with plane-parallel faces, of area $0.5-0.8 \mathrm{~cm}^{2}$, and thickness $0.4-0.8 \mathrm{~mm}$. The samples were polished with alumina powder using three sizes of grain 1.0, 0.5 , and $0.3 \mu \mathrm{m}$ in decreasing sequential order. Surface organic impurities were eliminated just before collecting the data using sequence solvents (tricloroetilen, acetone, and ethanol) in an ultrasonic bath, and finally the samples were blown dry with nitrogen.

Ellipsometry studies have demonstrated that the treatment of bulk semiconducting samples with mechanicalchemical polishing in an alkaline solution efficiently eliminates oxide layers on the surface, allowing enough time for measurements to be performed before the layer forms again. ${ }^{26-30}$ This procedure replaces the corrections that are made with numerical models to eliminate the effects due to the oxide overlayers. ${ }^{30,31}$ Therefore for our experiments, immediately before performing the ellipsometry measurements the samples were chemically polished with a colloidal suspension (Buehler Mastermet), ${ }^{26-30}$ rinsed in ethanol (5 min), and blown dry with nitrogen.

\section{OPTICAL MEASUREMENTS AND ANALYSIS METHODOLOGY}

The optical measurements were performed with a commercial ellipsometer (variable-angle spectroscopic ellipsometer J. A. Woollam) ${ }^{24}$ with a rotating polarizer, and an autoretarder that allows us to measure ellipsometric angle between $0^{\circ}$ and $360^{\circ}$, and thus to obtain accurate measurements in the spectral regions of small absorption. The ellipsometric spectra were measured at angles of incidence of $55^{\circ}$ and $65^{\circ}$ to ensure a consistent and accurate determination of the dielectric constant of the material. In order to assure an optimal signal, each sample was carefully aligned before performing each measurement.

In the present work the effects of a surface oxide layer have been diminished as previously explained, and the results can be assumed to be representative of the bulk material. Thus the two phase model (atmosphere sample) ${ }^{31}$ can be used to analyze the ellipsometry spectra and to determine the dielectric function.

According to the two phase model the optical parameters are related by the following expressions:

$$
\rho=r_{p} / r_{s}=(\tan \Psi)\left(e^{i \Delta}\right),
$$

$$
\begin{aligned}
\varepsilon(\omega) & =\varepsilon_{a}\left[\left(\frac{1-\rho}{1+\rho}\right)^{2} \sin ^{2} \phi \tan ^{2} \phi+\sin ^{2} \phi\right] \\
& =\left(n^{2}-k^{2}\right)+i(2 n k),
\end{aligned}
$$

where $\rho$ is the complex ratio of the Fresnel reflection coefficients $r_{p}$ and $r_{s}, \Psi$, and $\Delta$ are the ellipsometric angles, related to the amplitude and the difference of phase between the components parallel $s$ and perpendicular $p$ to the electric field of the polarized wave. $\varepsilon(\omega)=\varepsilon_{1}(\omega)+i \varepsilon_{2}(\omega)$ is the complex dielectric function of the sample, and $\varepsilon_{a} \equiv n_{0}^{2}$ is the dielectric function or the square refractive index of the atmosphere, $\phi$ is the incidence angle, $n$ is the complex refractive index, and $k$ is the extinction coefficient. The spectroscopic ellipsometric system measures the angles $\phi, \Psi, \Delta$ thus allowing us to calculate the optical properties $\varepsilon_{1}$ and $\varepsilon_{2}$, or equivalently $n$ and $k$.

The critical points (PCs) of the complex dielectric function $\varepsilon(\omega)$ are analyzed by means of the numerical calculation of the second derivative $\left(d^{2} \varepsilon_{1} / d \omega^{2}\right.$ and $\left.d^{2} \varepsilon_{2} / d \omega^{2}\right)$ from the experimental spectrum of the real $\varepsilon_{1}$ and imaginary $\varepsilon_{2}$ components. The first principal energy $E_{g}$ and the interband transitions for $h \nu \gg E_{g}$ were determined using a conventional analysis method ${ }^{29,30,32,33}$ that allows us to improve the resolution. The method consists of fitting the second derivative to standard analytic functions. These analytic expressions and the corresponding theoretical derivatives are given by

$$
\varepsilon(\omega)=C-A e^{i \theta}(\omega-E+i \gamma)^{m}
$$

for $m \neq 0$, and

$$
\frac{d^{2} \varepsilon}{d \omega^{2}}=-m(m-1) A e^{i \theta}(\omega-E+i \gamma)^{m-2} .
$$

For $m=0$, the dielectric function is

$$
\varepsilon(\omega)=C-A e^{i \theta} \ln (\omega-E+i \gamma)
$$

and

$$
\frac{d^{2} \varepsilon}{d \omega^{2}}=A e^{i \theta}(\omega-E+i \gamma)^{-2}
$$

where $A$ is the amplitude, $E$ it is the threshold energy, $\gamma$ is the broadening, and $\theta$ is the excitonic phase angle. These parameters are determined by fitting the numerically obtained second derivative spectra of the experimental dielectric function. According to the value of exponent $m$ in these expressions, the PCs are of the type: ${ }^{29}$ one-dimensional (1D) for $m=-\frac{1}{2}$, two-dimensional (2D) for $m=0$, threedimensional (3D) for $m=\frac{1}{2}$, and for the case of discrete excitons $m=-1$.

In order to apply numerically the Eqs. (4) and (6) it is necessary to express these in terms of its real and imaginary components as follows:

For $m \neq 0$ 


$$
\begin{aligned}
\frac{d^{2} \varepsilon}{d \omega^{2}}= & A^{\prime}(\Omega)^{(m-2) / 2}\left\{\cos \left[(m-2) \operatorname{arcos}\left(\frac{\omega-E}{\Omega^{1 / 2}}\right)+\theta\right]\right. \\
& \left.+i \sin \left[(m-2) \operatorname{arcos}\left(\frac{\omega-E}{\Omega^{1 / 2}}\right)+\theta\right]\right\},
\end{aligned}
$$

where $A^{\prime}=-m(m-1) A ; \Omega=(\omega-E)^{2}+\gamma^{2}$.

For $m=0$

$$
\begin{aligned}
\frac{d^{2} \varepsilon}{d \omega^{2}}= & \frac{A}{\Omega}\left\{\cos \left[-2 \operatorname{arcos}\left(\frac{\omega-E}{\Omega^{1 / 2}}\right)+\theta\right]\right. \\
& \left.+i \sin \left[-2 \operatorname{arcos}\left(\frac{\omega-E}{\Omega^{1 / 2}}\right)+\theta\right]\right\} .
\end{aligned}
$$

The symmetry and structure of the electronic bands of some ternary semiconductors chalcopyrite $(\mathrm{I}(\mathrm{Cu})-\mathrm{III}(\mathrm{Ga}, \mathrm{Al}$, In) $-\mathrm{VI}_{2}$ ) have been calculated by Jaffe and Zunger. ${ }^{34}$ In the simplest approach where only symmetric differences are considered, the electronic structure of I-III- $-\mathrm{VI}_{2}$ chalcopyrites can be derived from that of zinc blende (ZB). ${ }^{35,36} \mathrm{In}$ other works, according to the selection rules, it has been proposed, for a system similar to the one studied here, a scheme of energy levels and interband transitions $(\Gamma, N$ and $T)$ deduced from the dielectric function spectrum $\varepsilon(\omega) .{ }^{25-28}$ It has been established that in the electronic band structure, transitions $\Gamma$ are at center of the first Brillouin zone $(\mathrm{ZB})$, within these included the first energy gap $E_{g}$. Transitions $N$ and $T$ belong to the edges of the zone $(\mathrm{ZB})^{34}$ and therefore in the dielectric function spectrum these transitions are observed at energies higher than $E_{g}$. For this work in the following it will be assumed that the symmetries and ordering of the transitions of the system $\mathrm{Ag}_{1-x} \mathrm{Cu}_{x} \mathrm{InSe}_{2}$ are in agreement with the above described structure.

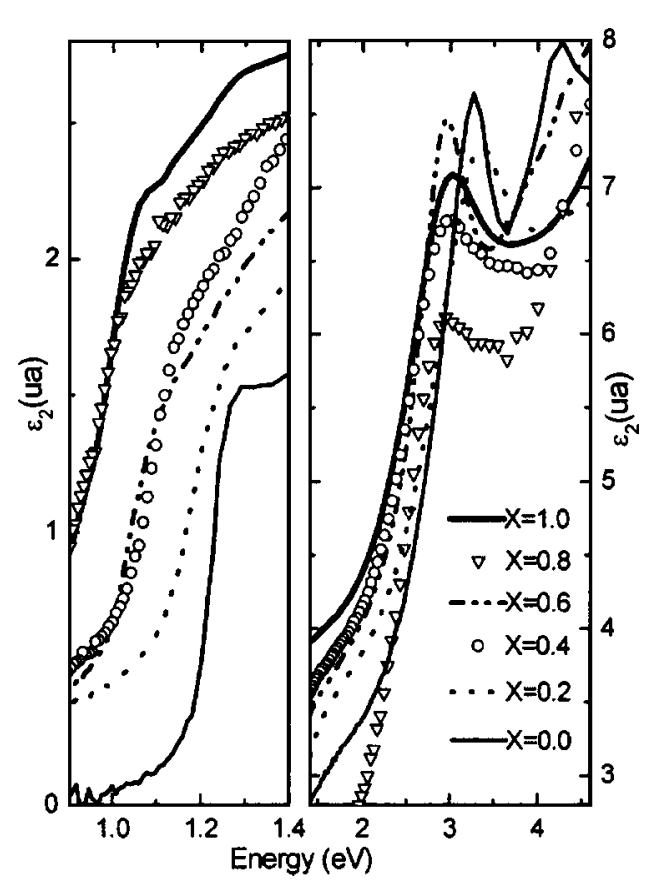

FIG. 1. Imaginary part of the dielectric function $\left(\epsilon_{2}\right)$ for $\mathrm{Ag}_{1-x} \mathrm{Cu}_{x} \mathrm{InSe}_{2}$ with $x=0.0,0.2,0.4,0.6,0.8$, and 1.0 .

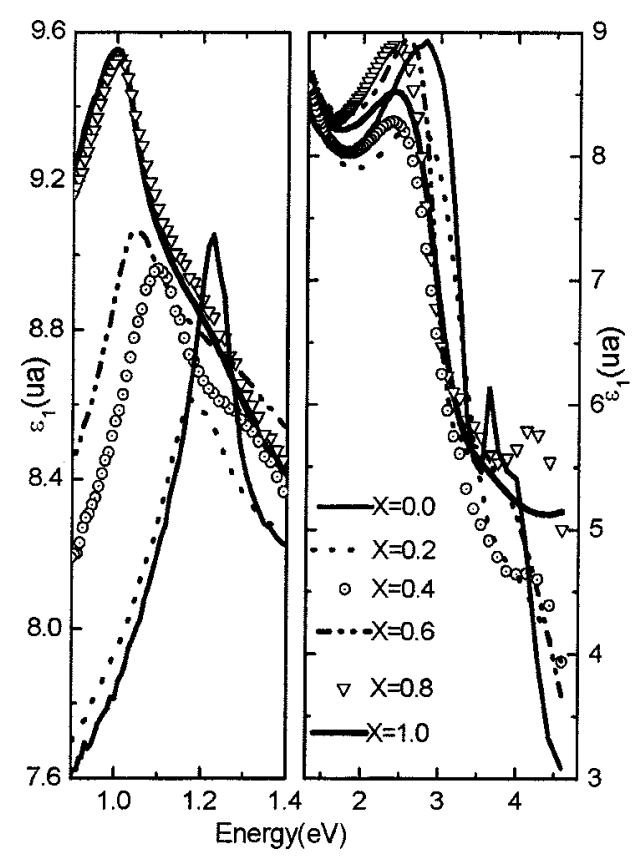

FIG. 2. Real part of the dielectric function $\left(\epsilon_{1}\right)$ for $\mathrm{Ag}_{1-x} \mathrm{Cu}_{x} \mathrm{InSe}_{2}$ with $x$ $=0.0,0.2,0.4,0.6,0.8$, and 1.0 .

\section{RESULTS AND DISCUSSION}

Table I contains a summary of the experimental values, obtained in the present work, of the refractive index $(n)$ and extinction coefficient $(k)$ in the energy interval $0.9-4.6 \mathrm{eV}$. The refractive indices values of $\mathrm{CuInSe}_{2}$ are in very good agreement (better than 5\%) with the values reported by Alonso et $a .^{28}$ for a single crystal. In the same way our extinction coefficient values agree with the $k_{\perp}$ values of Alonso et al.

Figures 1 and 2 show, respectively, experimental spectra of the imaginary $\varepsilon_{2}(\omega)$ and real $\varepsilon_{1}(\omega)$ components of the complex dielectric function $\varepsilon(\omega)$. Each spectrum is separated in two regions of energy in order to better observe the structure of these components, the near infrared (NIR) region $0.9-1.4 \mathrm{eV}$ and the $1.4-4.6 \mathrm{eV}$ region that includes the visible and near ultraviolet (NUV). These curves show edges and peaks that correspond to critical points (CPs) of energy transitions of the electronic band structure. ${ }^{26-33}$ In the NIR region the fundamental energy gap $E_{0}=E_{g}$ is distinguished for each sample, and in the region (1.4-4.6) $\mathrm{eV}$ appears a second energy threshold $E_{1}$. In addition to the main energy thresholds $\left(E_{g}, E_{1}\right)$, it is also possible to observe other small edges and peaks, with lower intensity and less definition, that correspond to other energy transitions. The precise values of the $E_{g}$ and $E_{1}$ energy thresholds and the energies for the transitions $\Gamma, N$, and $T$ have been determined by theoretical fitting of the second derivative with the expressions (7) and (8) as explained in Sec. III.

Figures 3 and 4 show the experimental spectrum of the second derivative with respect to the photon energy from the experimental components of the dielectric function $\left(d^{2} \varepsilon_{2} / d \omega^{2}\right.$ and $\left.d^{2} \varepsilon_{1} / d \omega^{2}\right)$. The theoretical fitting as determined by expressions (7) and (8) is also shown. The scale of energy has been divided into three regions. In the region of $0.9-1.25 \mathrm{eV}$ is found the energy threshold of the fundamen- 
TABLE I. Selected experimental values of the refractive index $n$ and extinction coefficient $k$ in the energy interval $(0.9-4.6) \mathrm{eV}$ for the $\mathrm{Ag}_{x-1} \mathrm{Cu}_{x} \mathrm{InSe}_{2}$ system, with $x=0.0,0.1,0.2,0.4,0.6,0.8,1.0$.

\begin{tabular}{|c|c|c|c|c|c|c|c|c|c|c|c|c|}
\hline \multirow[b]{3}{*}{$h v(\mathrm{eV})$} & \multicolumn{12}{|c|}{ Composition $x$} \\
\hline & 0 & 0.2 & 0.4 & 0.6 & 0.8 & 1.0 & 0 & 0.2 & 0.4 & 0.6 & 0.8 & 1.0 \\
\hline & \multicolumn{6}{|c|}{$N$} & \multicolumn{6}{|c|}{$K$} \\
\hline 4.592 & 2.342 & 2.386 & 2.455 & 2.438 & 2.654 & 2.592 & 1.549 & 1.320 & 1.445 & 1.509 & 1.396 & 1.255 \\
\hline 4.428 & 2.389 & 2.411 & 2.492 & 2.486 & 2.845 & 2.570 & 1.543 & 1.296 & 1.348 & 1.434 & 1.316 & 1.218 \\
\hline 4.275 & 2.502 & 2.435 & 2.489 & 2.532 & 2.826 & 2.558 & 1.516 & 1.271 & 1.261 & 1.374 & 1.210 & 1.189 \\
\hline 4.133 & 2.615 & 2.461 & 2.466 & 2.559 & 2.779 & 2.555 & 1.421 & 1.246 & 1.197 & 1.300 & 1.158 & 1.166 \\
\hline 3.999 & 2.663 & 2.479 & 2.454 & 2.581 & 2.738 & 2.558 & 1.299 & 1.226 & 1.175 & 1.247 & 1.129 & 1.150 \\
\hline 3.874 & 2.647 & 2.497 & 2.456 & 2.600 & 2.726 & 2.567 & 1.239 & 1.222 & 1.169 & 1.203 & 1.104 & 1.139 \\
\hline 3.757 & 2.652 & 2.524 & 2.479 & 2.594 & 2.732 & 2.579 & 1.185 & 1.225 & 1.166 & 1.164 & 1.095 & 1.129 \\
\hline 3.647 & 2.716 & 2.559 & 2.501 & 2.617 & 2.727 & 2.592 & 1.116 & 1.238 & 1.160 & 1.133 & 1.068 & 1.123 \\
\hline 3.542 & 2.617 & 2.625 & 2.523 & 2.617 & 2.749 & 2.606 & 1.178 & 1.238 & 1.150 & 1.098 & 1.078 & 1.122 \\
\hline 3.444 & 2.656 & 2.689 & 2.545 & 2.619 & 2.759 & 2.623 & 1.222 & 1.243 & 1.145 & 1.089 & 1.075 & 1.126 \\
\hline 3.351 & 2.749 & 2.768 & 2.577 & 2.613 & 2.776 & 2.647 & 1.272 & 1.221 & 1.144 & 1.100 & 1.070 & 1.135 \\
\hline 3.263 & 2.900 & 2.836 & 2.625 & 2.643 & 2.802 & 2.678 & 1.238 & 1.179 & 1.132 & 1.112 & 1.060 & 1.145 \\
\hline 3.179 & 2.996 & 2.889 & 2.652 & 2.674 & 2.818 & 2.719 & 1.163 & 1.120 & 1.133 & 1.148 & 1.066 & 1.150 \\
\hline 3.100 & 3.063 & 2.931 & 2.691 & 2.730 & 2.858 & 2.768 & 1.065 & 1.055 & 1.135 & 1.175 & 1.058 & 1.146 \\
\hline 3.024 & 3.087 & 2.959 & 2.741 & 2.810 & 2.893 & 2.820 & 0.966 & 0.992 & 1.124 & 1.186 & 1.051 & 1.131 \\
\hline 2.883 & 3.086 & 2.978 & 2.839 & 2.984 & 2.970 & 2.919 & 0.815 & 0.874 & 1.070 & 1.110 & 1.021 & 1.065 \\
\hline 2.818 & 3.082 & 2.978 & 2.883 & 3.050 & 3.009 & 2.956 & 0.754 & 0.826 & 1.029 & 1.031 & 0.987 & 1.020 \\
\hline 2.695 & 3.051 & 2.968 & 2.942 & 3.095 & 3.069 & 3.002 & 0.657 & 0.737 & 0.933 & 0.869 & 0.906 & 0.922 \\
\hline 2.638 & 3.037 & 2.961 & 2.958 & 3.091 & 3.086 & 3.011 & 0.619 & 0.701 & 0.887 & 0.805 & 0.864 & 0.875 \\
\hline 2.583 & 3.022 & 2.950 & 2.968 & 3.083 & 3.099 & 3.016 & 0.583 & 0.670 & 0.838 & 0.747 & 0.816 & 0.831 \\
\hline 2.530 & 3.008 & 2.939 & 2.971 & 3.070 & 3.107 & 3.017 & 0.554 & 0.644 & 0.795 & 0.700 & 0.772 & 0.792 \\
\hline 2.480 & 2.992 & 2.930 & 2.969 & 3.055 & 3.109 & 3.014 & 0.525 & 0.619 & 0.756 & 0.663 & 0.731 & 0.757 \\
\hline 2.384 & 2.963 & 2.911 & 2.959 & 3.026 & 3.104 & 3.001 & 0.489 & 0.580 & 0.693 & 0.603 & 0.658 & 0.699 \\
\hline 2.296 & 2.941 & 2.892 & 2.943 & 3.000 & 3.084 & 2.985 & 0.451 & 0.549 & 0.643 & 0.561 & 0.608 & 0.652 \\
\hline 2.214 & 2.919 & 2.878 & 2.929 & 2.978 & 3.068 & 2.968 & 0.429 & 0.525 & 0.605 & 0.528 & 0.555 & 0.616 \\
\hline 2.101 & 2.894 & 2.862 & 2.907 & 2.953 & 3.034 & 2.949 & 0.401 & 0.497 & 0.564 & 0.493 & 0.514 & 0.575 \\
\hline 2.000 & 2.877 & 2.852 & 2.891 & 2.936 & 3.010 & 2.932 & 0.378 & 0.476 & 0.534 & 0.466 & 0.476 & 0.546 \\
\hline 1.907 & 2.868 & 2.847 & 2.880 & 2.924 & 2.987 & 2.920 & 0.362 & 0.458 & 0.515 & 0.446 & 0.455 & 0.525 \\
\hline 1.797 & 2.859 & 2.847 & 2.875 & 2.918 & 2.968 & 2.911 & 0.345 & 0.436 & 0.492 & 0.424 & 0.434 & 0.505 \\
\hline 1.698 & 2.860 & 2.852 & 2.876 & 2.916 & 2.957 & 2.907 & 0.324 & 0.417 & 0.475 & 0.403 & 0.420 & 0.490 \\
\hline 1.610 & 2.861 & 2.860 & 2.882 & 2.918 & 2.953 & 2.909 & 0.307 & 0.397 & 0.456 & 0.386 & 0.408 & 0.479 \\
\hline 1.590 & 2.856 & 2.863 & 2.884 & 2.920 & 2.954 & 2.910 & 0.309 & 0.392 & 0.453 & 0.383 & 0.407 & 0.476 \\
\hline 1.494 & 2.869 & 2.882 & 2.896 & 2.928 & 2.955 & 2.920 & 0.284 & 0.360 & 0.436 & 0.363 & 0.403 & 0.463 \\
\hline 1.409 & 2.879 & 2.891 & 2.920 & 2.941 & 2.964 & 2.934 & 0.264 & 0.326 & 0.410 & 0.340 & 0.390 & 0.452 \\
\hline 1.305 & 2.912 & 2.903 & 2.946 & 2.960 & 2.979 & 2.963 & 0.253 & 0.289 & 0.365 & 0.308 & 0.378 & 0.438 \\
\hline 1.204 & 2.989 & 2.937 & 2.960 & 2.975 & 3.002 & 2.998 & 0.089 & 0.217 & 0.315 & 0.263 & 0.354 & 0.402 \\
\hline 1.107 & 2.872 & 2.887 & 3.002 & 2.997 & 3.025 & 3.029 & 0.024 & 0.110 & 0.235 & 0.215 & 0.319 & 0.363 \\
\hline 1.097 & 2.863 & 2.879 & 3.002 & 3.001 & 3.027 & 3.033 & 0.020 & 0.103 & 0.218 & 0.207 & 0.319 & 0.361 \\
\hline 1.000 & 2.795 & 2.818 & 2.927 & 2.985 & 3.068 & 3.102 & 0.012 & 0.078 & 0.116 & 0.090 & 0.261 & 0.264 \\
\hline 0.905 & 2.761 & 2.777 & 2.865 & 2.910 & 3.058 & 3.046 & 0.006 & 0.065 & 0.090 & 0.057 & 0.159 & 0.150 \\
\hline
\end{tabular}

tal absorption edge $E_{0}=E_{g}$. This energy is identified in the spectrum of the second numerical derivative by a sharp and intense peak, and is related to an electronic transition of $\Gamma$ type. This threshold $E_{g}$ corresponds to direct transition from the valence band maximum to the conduction band minimum. In the region of $2.5-4.6 \mathrm{eV}$ a second energy threshold $E_{1}$ that has been related to a $N$ type transition appears. In the same region but with very low intensity other possible transitions of $T$ type were observed. In the intermediate region $(1.25-2.40) \mathrm{eV}$ appear a series of peaks very close one to each other, which have been attributed to transitions of the $\Gamma$ and $N$ types. The intensity of these peaks is very low, so they are difficult to evaluate in the second derivative spectra due to the high intensity of the transitions with $E_{0}$ and $E_{1}$ energies. Thus it has been necessary to separate the intermediate scale and to magnify it by a factor of 10 with respect to that of the other regions.

The fits of Figs. 3 and 4 have been obtained considering CPs of the three-dimensional type $3 \mathrm{D}$ in the $E_{g}$ region (NIR), with values of $m=1 / 2, A>0$, and $\phi>0$, and in the $E_{1}$ region (NUV) with PCs of the type 2D with values $m=-1 / 2, A$ $>0$, and $\phi>0$.

Table II contains the average values of the electronic transitions $E_{g}, E_{1}, \Gamma, N$, and $T$ obtained from the second derivative, for the compositions studied. These results are in good agreement with those reported for $\operatorname{CIS}^{26,27,37}(x=1)$ and 


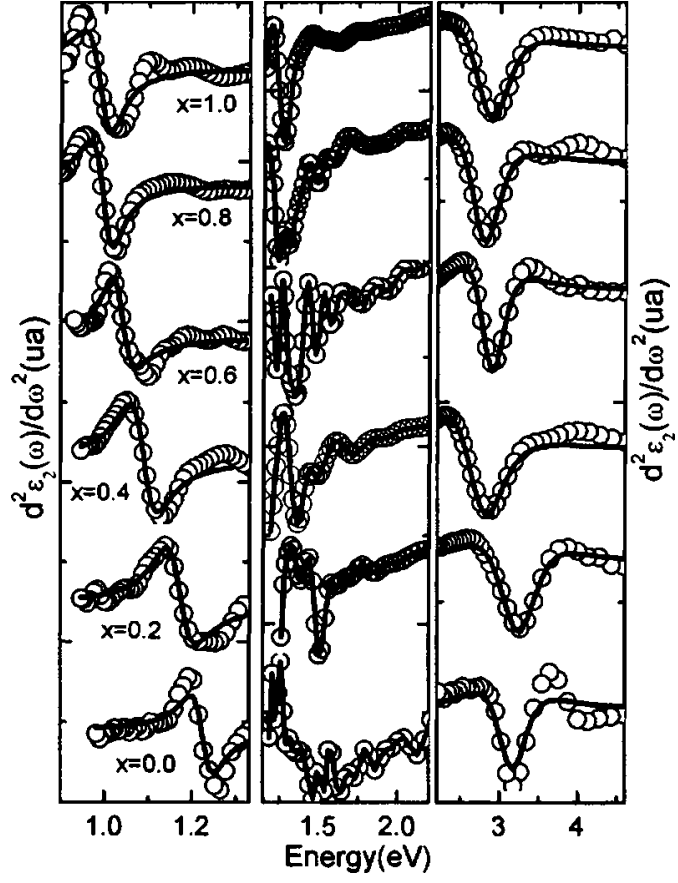

FIG. 3. Second numerical derivative from the imaginary component of the dielectric function $\epsilon_{2}$ and the theoretical fitting in the energy regions of $E_{g}$ and $E_{l}$. For clarity the scale of the central plot has been magnified by a factor of 10 .

with the $E_{g}$ value reported for AIS ${ }^{9-11,38,37}(x=0)$. Following the notation of the energy levels of Table II, Fig. 5 shows a schematic representation of the energy levels and the transitions $\Gamma, N$, and $T$, that contribute to the measured dielectric function for the semiconductor system $\mathrm{Ag}_{1-x} \mathrm{Cu}_{x} \mathrm{InSe}_{2}$.

In Fig. 6 the transition energies of Table II $\left(\Gamma, N, T, E_{0}\right.$, and $E_{1}$ ) have been plotted as a function of the composition $x$. Is worth noting that for all the transitions studied there are two regions as a function of the composition that show a different behavior. For $x \leqslant 0.4$ there is a clear decrease of the energy as $x$ increases, and for $x \geqslant 0.6$ the energy variation is weaker.

In order to better analyze the behavior of the main energy thresholds, the energies $E_{g}=E_{0}$ and $E_{1}$ have been plotted as a function of the composition $x$ in Fig. 7. In both curves are observed two different behaviors. For $x \leqslant 0.4$, the nearest region to the dominion of $n$-AIS, the $E_{0}$ and $E_{1}$ en-

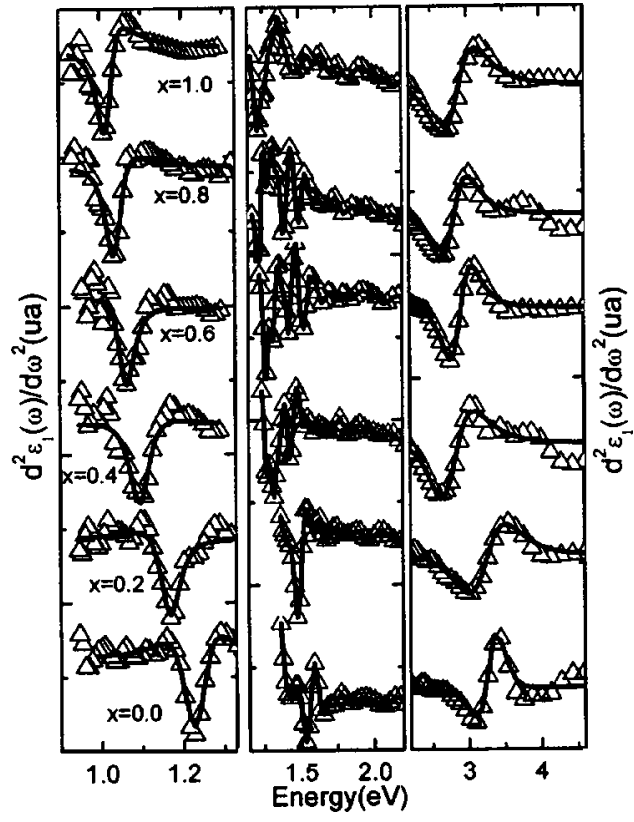

FIG. 4. Second numerical derivative from the real component $\epsilon_{2}$ and the theoretical fitting in the energy regions of $E_{g}$ and $E_{l}$. For clarity the scale of the central plot has been magnified by a factor of 10 .

ergies decrease remarkably with $x$, according to the expressions $\left(E_{g}=-0.33 x+1.228\right)$ and $\left(E_{1}=-5.425 x^{2}+x+3.214\right)$. While for to $x \geqslant 0.6$, the nearest region to the dominion of $p$-CIS the energies decrease smoothly according to the polynomials $\left(E_{g}=0.225 x^{2}-0.415 x+1.199\right)$ and $\left(E_{1}=1.95 x^{2}\right.$ $-3.23 x+4.101)$. These analytical expressions provide a suitable tool to evaluate the band-gap energies of $\mathrm{Ag}_{1-x} \mathrm{Cu}_{x} \mathrm{InSe}_{2}$ polycrystalline alloys with $x \leqslant 0.4$ and $x \geqslant 0.6$.

The behavior of the energy band gap $E_{g}=E_{0}$ values as a function of the atomic composition $x$ reported by Basewicz et al. ${ }^{15}$ using photoconductivity measurements $\left(E_{g}=0.305 x^{2}\right.$ $-0.571 x+1.278$ ) for polycrystalline $\mathrm{Ag}_{1-x} \mathrm{Cu}_{x} \mathrm{InSe}_{2}$ alloys, agree well with our results for $x>0.6$, but show significant discrepancies for $x<0.4$. Furthermore, for $x=0$ they find for the main energy gap $E_{g}=1.278 \mathrm{eV}$, which is far from the value determined in this work and also far from the energy gap usually reported of AIS. ${ }^{9-11,38,37}$ It is also interesting to note that in the case of thin films, values of the energy gaps have been reported for the composition $p-\mathrm{Ag}_{0.5} \mathrm{Cu}_{0.5} \operatorname{InSe}_{2}{ }^{13}$

TABLE II. Transition energies for the $\mathrm{Ag}_{x-1} \mathrm{Cu}_{x} \mathrm{InSe}_{2}$ system: $E_{g}=E_{0}=\Gamma_{4 V} \rightarrow \Gamma_{1 C} E_{1}=N_{1 V}^{(1)} \rightarrow N_{1 C}^{(2)}$

\begin{tabular}{lcccccc}
\hline \hline & \multicolumn{7}{c}{ Composition $x$} \\
\cline { 2 - 7 } \multicolumn{1}{c}{$\begin{array}{c}\text { Energy } \\
\text { transitions }(\mathrm{eV})\end{array}$} & 0.0 & 0.2 & 0.4 & 0.6 & 0.8 & 1.0 \\
\hline$\Gamma_{4 V} \rightarrow \Gamma_{1 C}$ & 1.225 & 1.168 & 1.093 & 1.031 & 1.011 & 1.009 \\
$\Gamma_{5 V}^{(1)} \rightarrow \Gamma^{1 C}(B)$ & 1.474 & 1.329 & 1.241 & 1.203 & 1.221 & 1.192 \\
$\Gamma_{5 V}^{(1)} \rightarrow \Gamma_{1 C}(C)$ & 1.647 & 1.546 & 1.435 & 1.440 & 1.374 & 1.368 \\
$\Gamma_{4 V}^{(1)} \rightarrow \Gamma_{3 C}$ & 1.797 & 1.772 & 1.600 & 1.634 & 1.573 & 1.550 \\
$\Gamma_{5 V}^{(1)} \rightarrow \Gamma_{3 C}$ & 1.969 & 1.957 & 1.843 & 1.861 & 1.734 & 1.708 \\
$N_{1 V}^{(1)} \rightarrow N_{1 V}^{(1)}$ & 2.138 & 2.175 & 2.103 & 2.032 & 1.923 & 1.937 \\
$\Gamma_{5 V}^{(2)} \rightarrow \Gamma_{1 C}$ & 2.318 & 2.450 & 2.296 & 2.175 & 2.102 & 2.214 \\
$N_{1 V}^{(1)} \rightarrow N_{1 C}^{(2)}$ & 3.214 & 3.197 & 2.746 & 2.865 & 2.765 & 2.821 \\
$T_{V} \rightarrow T_{C}$ & 4.428 & 4.352 & 3.999 & 4.510 & 3.878 & 4.500 \\
\hline \hline
\end{tabular}




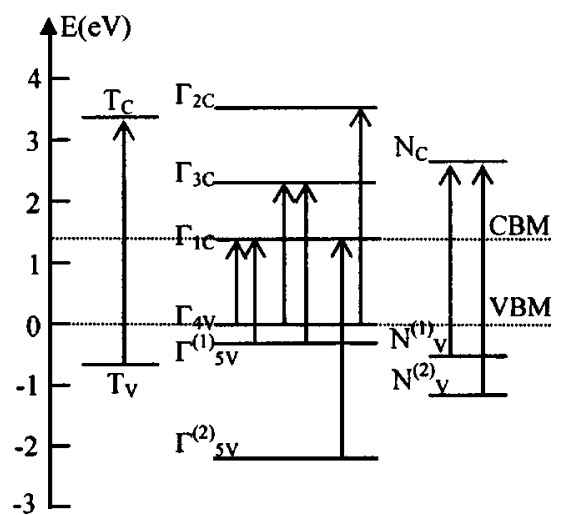

FIG. 5. Scheme of electronic-energy levels and the transitions of $\mathrm{Ag}_{1-x} \mathrm{Cu}_{x} \mathrm{InSe}_{2}$. The symmetries are given in single group notation.

The values have been determined using optical absorption and are in good agreement with our results of Table II, for $0.4<x<0.6$.

The lattice symmetry influences the electronic bands of the ternary chalcopyrite systems I-III- $\mathrm{VI}_{2}$, so the deviations of the lattice parameters from its tetragonal ideal values induce the splitting in the upper edges of the valence band. ${ }^{34}$ The $d$ levels that the metals $\mathrm{I}(\mathrm{Cu}, \mathrm{Ag})$ bring to the chalcopyrite structure build the valence bands $\Gamma$, and the proximity of these cations with the anions VI (Se) also modify the splitting of these bands. In the present work, the tetragonal deviation ${ }^{25,37} \eta-1$ of AIS $(\eta \equiv c / 2 a=0.959)$ is larger than in CIS $(\eta=1.004)$, and as a consequence this fact produces higher crystalline field $\Delta_{\text {cr }}$ and thus the hybridization of $d$ levels in AIS will be greater than in CIS. This aspect contributes to explaining the different behaviors found in the transition energies from the system $\mathrm{Ag}_{1-x} \mathrm{Cu}_{x} \mathrm{InSe}_{2}$, whose properties are described according to composition $x$ either in the dominion of $n$-AIS, or of $p$-CIS.

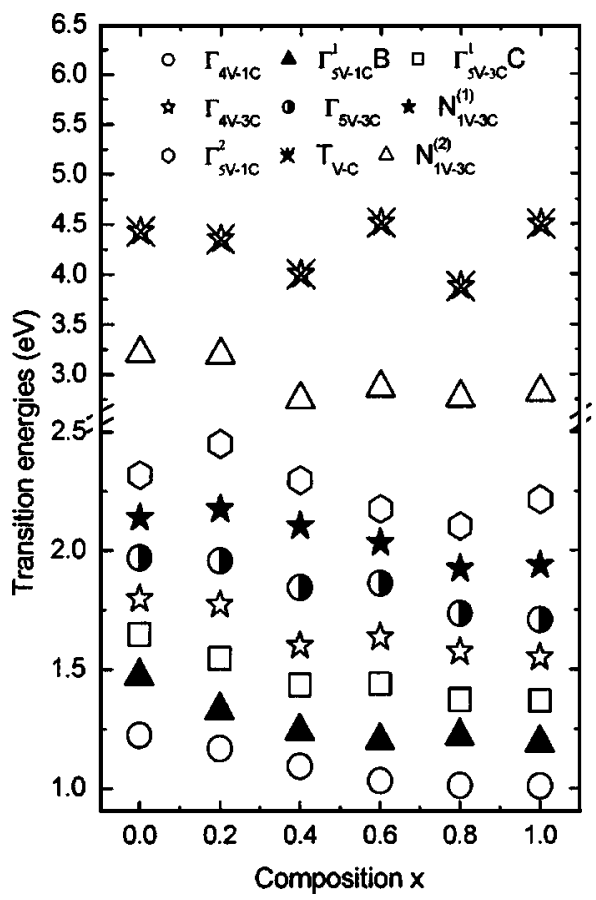

FIG. 6. Energies estimated for the electronic transitions, as a function of the composition $x$ for the system $\mathrm{Ag}_{1-x} \mathrm{Cu}_{x} \mathrm{InSe}_{2}$.

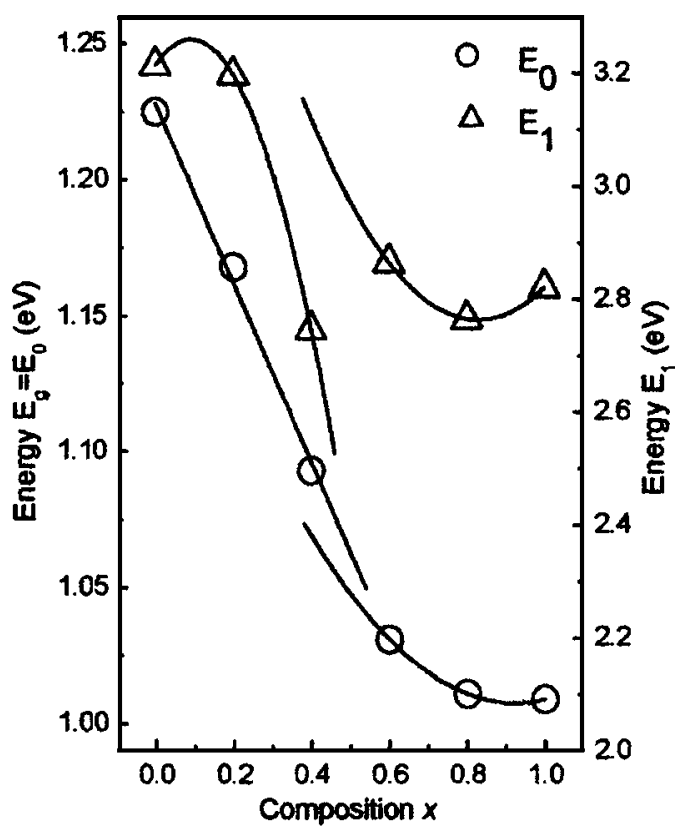

FIG. 7. Energies $E_{0}$ and $E_{1}$ as a function of the composition $x$ of $\mathrm{Ag}_{1-x} \mathrm{Cu}_{x} \mathrm{InSe}_{2}$, calculated by fitting the second numerical derivative of $\epsilon_{1}$ and $\epsilon_{2}$. The lines are fits to analytical polynomial.

\section{CONCLUSIONS}

The dielectric function has been determined for the whole composition range $x$ of the $\mathrm{Ag}_{1-x} \mathrm{Cu}_{x} \mathrm{InSe}_{2}$ system. The analysis of the dielectric function has allowed us to identify and evaluate the energy of the electronic transitions $\Gamma, N, T, E_{0}$, and $E_{1}$. The value of the fundamental energy gap $E_{g}$ in the system changes from 1.225 to $1.009 \mathrm{eV}$ when the material is transformed from $n$-AIS to $p$-CIS, by means of the ordered substitution of $\mathrm{Ag}$ by $\mathrm{Cu}$ in the cation lattice.

The behavior of the transition energies as a function of the composition $x$ shows two clear differentiated regimes: for $x \leqslant 0.4$ and for $x \geqslant 0.6$. In the first gap $E_{g}$ for $x \leqslant 0.4$ decays linearly and for $x \geqslant 0.6$ the evolution is weaker and can be approximately fitted by a second order polynomial. The origin of these different behaviors has been discussed in terms of the different structural properties of $n$-AIS and $p$-CIS materials. The present results offer a valuable set of data over a large range of compositions that can be useful for the design of high efficiency solar cells based in the $\mathrm{Ag}_{1-x} \mathrm{Cu}_{x} \mathrm{InSe}_{2}$ system.

\section{ACKNOWLEDGMENTS}

This study has been made by the economic support granted by the $\mathrm{CDCH}-\mathrm{UC}$, Valencia Venezuela, and was partially funded by CICYT (Spain), under Grant No. MAT200301490 and INTAS Project No. 03-6314.

${ }^{1}$ M. A. Green, K. Emery, D. L. King, S. Igari, and W. Warta, Prog. Photovoltaics 12, 55 (2004).

${ }^{2}$ K. Ramanathan et al., Prog. Photovoltaics 11, 225 (2003).

${ }^{3}$ H. W. Schock, Sol. Energy Mater. Sol. Cells 34, 19 (1994).

${ }^{4}$ J. M. Merino, M. León, F. Rueda, and R. Díaz, Thin Solid Films 22, 361 (2000).

${ }^{5}$ S. M. Wasim, Sol. Cells 16, 289 (1986).

${ }^{6}$ J. G. Albornoz, S. M. Wasim, and C. Rincón, Cryst. Res. Technol. 34, 1191 (1999) 
${ }^{7}$ J. M. Merino, J. L. Martin, S. Mahanty, R. Diaz, F. Rueda, and M. León, J. Appl. Phys. 80, 5610 (1996).

${ }^{8}$ K. Bindu, C. Sudha Kartha, K. P. Vijayakumar, T. Abe, and Y. Kashiwaba, Sol. Energy Mater. Sol. Cells 79, 67 (2003).

${ }^{9}$ J. L. Shay, B. Tell, H. M. Kasper, and L. M. Schiavone, Phys. Rev. B 7, 4485 (1973).

${ }^{10}$ A. El-Korashy, M. A. Abdel-Rahim, and H. El-Zahed, Thin Solid Films 338, 207 (1999).

${ }^{11}$ Y. Ueno, Y. Kojima, T. Sugiera, and H. Minoura, Thin Solid Films 189, 91 (1990).

${ }^{12}$ G. Venkata Rao, G. Hema Chandra, O. M. Hussain, S. Uthanna, and B. Srinivasulu Naidu, J. Alloys Compd. 325, 12 (2001).

${ }^{13}$ G. Venkata Rao, G. Hema Chandra, P. Sreedhara Reddy, O. M. Hussain, K. T. Ramakrisha, and S. Uthanna, Vacuum 67, 293 (2002).

${ }^{14}$ G. Dagan, T. F. Ciszek, and D. Cahen, J. Phys. Chem. 96, 11009 (1992).

${ }^{15}$ R. Bacewicz, J. R. Durrant, T. F. Ciszek, and S. K. Deb, Proceedings of the 7th International Conference on Ternary and Multinary Compounds, edited by S. K. Deb and A. Zunger (Materials Research Society, Pittsburgh, PA, 1987), p. 155.

${ }^{16}$ V. F. Gremenok, I. V. Bodnar, I. Martil, F. L. Martines, S. L. Sergeev Nekrasov, and I. A. Victorov, Solid State Phenom. B6/7, 361 (1999).

${ }^{17}$ T. F. Ciszek, J. Cryst. Growth 79, 689 (1986).

${ }^{18}$ J. E. Avon, K. Yoodee, and J. C. Woolley, J. Appl. Phys. 55, 524 (1984)

${ }^{19}$ T. Tinoco, M. Quintero, and C. Rincón, Phys. Rev. B 44, 1613 (1991).

${ }^{20}$ Y. Kenji, M. Naoji, S. Mutsumi, F. C. Shigefusa, K. Hironori, and I. Tetsuo, Physica B 302, 349 (2001).

${ }^{21}$ M. E. Beck, T. Weiss, D. Fisher, S. Fiecher, A. Jäger-Waldau, and M. Ch. Lux S., Thin Solid Films 361, 130 (2000).

${ }^{22}$ I.-H. Choi and P. Y. Yu, Phys. Status Solidi B 223, 313 (2001).
${ }^{23}$ I.-H. Choi, S.-H. Eom, and P. Y. Yu, J. Appl. Phys. 87, 3815 (2000).

${ }^{24}$ J. A. Woollam, in "Ellipsometry, variable angle spectroscopic," Wiley Encyclopedia of Electrical and Electronics Engineering, edited by J. G. Weber (Wiley, New York, 2000).

${ }^{25}$ J. G. Albornoz, Ph.D. thesis, Universidad Autónoma de Madrid, Madrid, Spain, 2005.

${ }^{26}$ M. I. Alonso, M. Garriga, C. A. Durante Rincón, and M. León, J. Appl. Phys. 88, 5796 (2000).

${ }^{27}$ K. Takahiro, A. Sadao, M. Hideto, and S. Koichi, J. Appl. Phys. 84, 5202 (1998).

${ }^{28}$ M. I. Alonso, K. Wakita, J. Pascual, M. Garriga, and N. Yamamoto, Phys. Rev. B 63, 075203 (2001).

${ }^{29}$ P. Lautenschlager, M. Garriga, S. Logothetidis, and M. Cardona, Phys. Rev. B 35, 9174 (1987).

${ }^{30}$ D. E. Aspnes and A. A. Studna, Phys. Rev. B 27, 985 (1983).

${ }^{31}$ H. G. Tompkins and W. A. McGahan, Spectroscopic Ellipsometry and Reflectometry (Wiley, New York, 1999).

${ }^{32}$ J. T. Benhlal, K. Strauch, R. Granger, and R. Triboulet, Opt. Mater. (Amsterdam, Neth.) 12, 143 (1999).

${ }^{33}$ L. Viña, S. Logothetidis, and M. Cardona, Phys. Rev. B 30, 1979 (1984).

${ }^{34}$ J. E. Jaffe and A. Zunger, Phys. Rev. B 29, 1882 (1984).

${ }^{35}$ J. Camassel, L. Artus, and J. Pascual, Phys. Rev. B 41, 5717 (1990).

${ }^{36}$ L. Artus, J. Pujol, and J. Pascual, Phys. Rev. B 41, 5727 (1990).

${ }^{37}$ J. L. Shay and H. Wernick, Ternary and Chalcopyrite Semiconductors: Growth, Electronic Properties and Applications (Pergamon, Oxford, 1975), pp. 4, 5, 118.

${ }^{38}$ L. Martinez, S. A. Lopez Rivera, and V. Sagredo, Nuovo Cimento D 2D, 1687 (1983). 